\title{
Mutual-Coupling Reduction in Metamaterial Substrate Integrated Waveguide Slotted Antenna Arrays Using Metal Fence Isolators for SAR and MIMO Applications
}

\author{
Mohammad Alibakhshikenari ${ }^{1 *}$, Bal S. Virdee ${ }^{2}$, Chan H. See ${ }^{3}$, Raed Abd-Alhameed ${ }^{4}$, Francisco Falcone ${ }^{5}$, and \\ Ernesto Limiti ${ }^{1}$ \\ ${ }^{1}$ Electronics Engineering Department, University of Rome "Tor Vergata", Via del Politecnico 1, 00133, Rome, \\ ITALY \\ ${ }^{2}$ London Metropolitan University, Center for Communications Technology, School of Computing \& Digital \\ Media, London N7 8DB, UK \\ ${ }^{3}$ School of Engineering, University of Bolton, Deane Road, Bolton, BL3 5AB, UK \\ ${ }^{4}$ School of Electrical Engineering \& Computer Science, University of Bradford, UK \\ ${ }^{5}$ Electric and Electronic Engineering Department, Universidad Pública de Navarra, SPAIN \\ *alibakhshikenari@ing.uniroma2.it
}

\begin{abstract}
A new type of mutual coupling reduction technique is applied to metamaterial substrate integrated waveguide (SIW) slotted antenna array. The circular shaped reference SIW antenna array is constructed from Alumina substrate with dimensions of $40 \times 5 \times 1.5 \mathrm{~mm}^{3}$. Embedded in the reference antenna are 38 slots with dimensions of $2 \times 1 \times 1.5 \mathrm{~mm}^{3}$. The reference SIW antenna operates over $\mathrm{X}$ - to $\mathrm{Ku}$-bands with average isolation between the radiation slots of approximately $-10 \mathrm{~dB}$. Isolation was increased by inserting metal fence isolators (MFIs) between the radiation slots, which increased the isolation by an average of $13 \mathrm{~dB}$. In addition, the antenna's impedance matching bandwidth is improved with no degradation in the radiation patterns. With MFIs the maximum gain achieved improves by $\sim \mathbf{1 0} \%$. The technique is simple to implement and proposed for synthetic aperture radar (SAR) and multiple input multiple output (MIMO) applications.
\end{abstract}

\section{INTRODUCTION}

Substrate integrated waveguide (SIW) is becoming popular in cavity-backed slot antenna designs as it has advantages of low cost, low-loss, easy fabrication and integration with planar circuits [1],[2]. In addition, SIW based array antennas have been shown to achieve high gain [3]. This is because: (i) SIW cavities functioning as slot radiators eliminate the need of feed network [4]; (ii) feed networks based on SIW have lower loss than those built using microstrips [5]; and (iii) isolation between array elements is improved with the closed-form SIW structure [6]. These attributes significantly reduce the complexity of array synthesis. Compared with other forms of antennas like bow-tie antennas, the limitation of slot antennas is their narrow bandwidth [7].

To overcome the bandwidth limitation and increase the isolation between the radiation slots, a SIW slotted antenna array (SIWSAA) design is proposed in this paper which is based on metamaterial technology [8]. The left-handed capacitance is shown to increase the bandwidth of SIWSAA without inflating its dimensions [9]. Furthermore, a novel technique is proposed to suppress the mutual coupling between the radiation elements with inclusion of metal fence isolators (MFIs) between the radiation elements.

\section{High ISOlation METAMATERIAL SIW SLOTTED ANTENNA ARRAY}

The reference metamaterial SIW slotted antenna array is shown in Fig. 1, where the slots essentially represent left-handed capacitance $\left(C_{L}\right)$ and inductance $\left(L_{L}\right)$ properties. This structure requires no metallic via-holes. Unwanted currents that flow over the waveguide structure result in voltage potential difference between the waveguide and the ground-plane. Hence, the waveguide structure can be represented by series right-handed inductance $\left(L_{R}\right)$ and shunt right-handed capacitance $\left(C_{R}\right)$. The 38 slotted antenna is a circular waveguide that was constructed with Alumina substrate with a high dielectric constant $\left(\varepsilon_{r}\right)$ of 9.9. Dimensions of the SIWSAA and the slots are: $40 \times 5 \times 1.5 \mathrm{~mm}^{3}$ and $2 \times 1 \times 1.5 \mathrm{~mm}^{3}$, respectively. 
S-parameter characteristics, reflection and transmission coefficients, of the reference SIWSAA are shown in Fig. 2. It is evident from these results the antenna covers six frequency bands from $\mathrm{X}$ - to Ku-bands with a maximum and average isolation between the radiation slots of approximately $-20 \mathrm{~dB}$ and $-10 \mathrm{~dB}$, respectively. To increasing the isolation between the radiation elements, MFIs were inserted between the radiation slots. This approach is shown to significantly improve reduction in mutual coupling between the radiation elements. The maximum, minimum, and average improvement in the isolation with MFIs are $20 \mathrm{~dB}, 8 \mathrm{~dB}$, and $13 \mathrm{~dB}$, respectively.

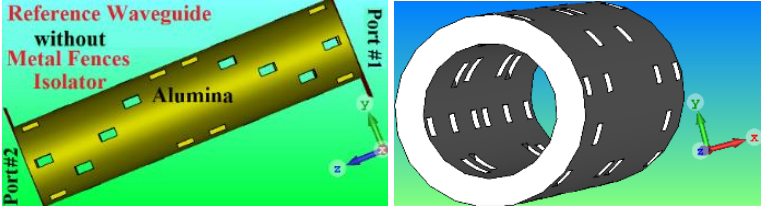

(a) Reference SIWSAA without MFIs (top and front views)
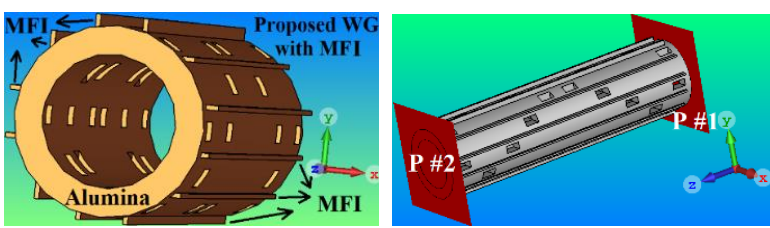

(b) Proposed SIWSAA with MFIs

Fig. 1. Configurations of the reference SIWSAA and the proposed SIWSAA with MFIs.
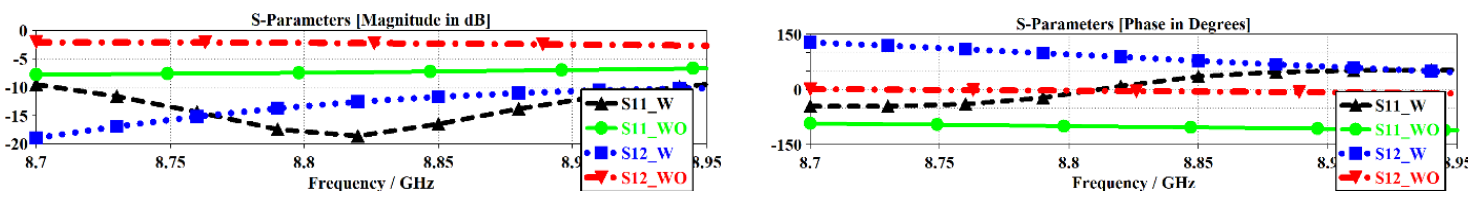

(a) First band
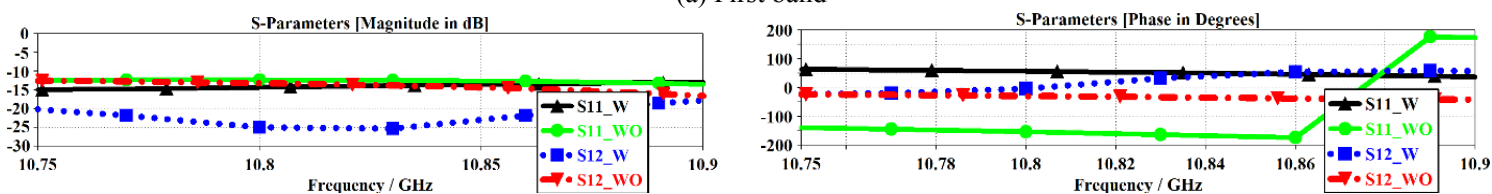

(b) Second band
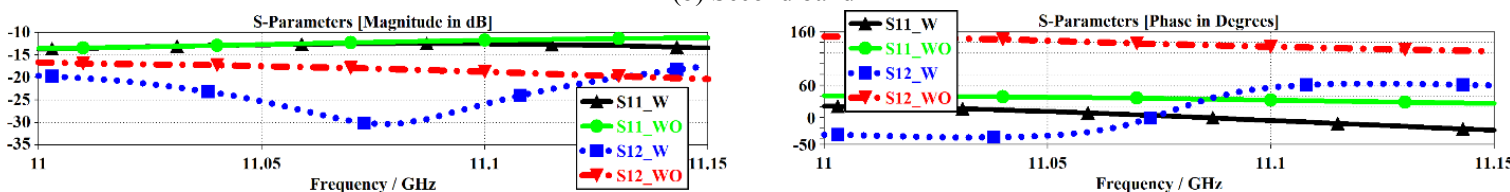

(c) Third band
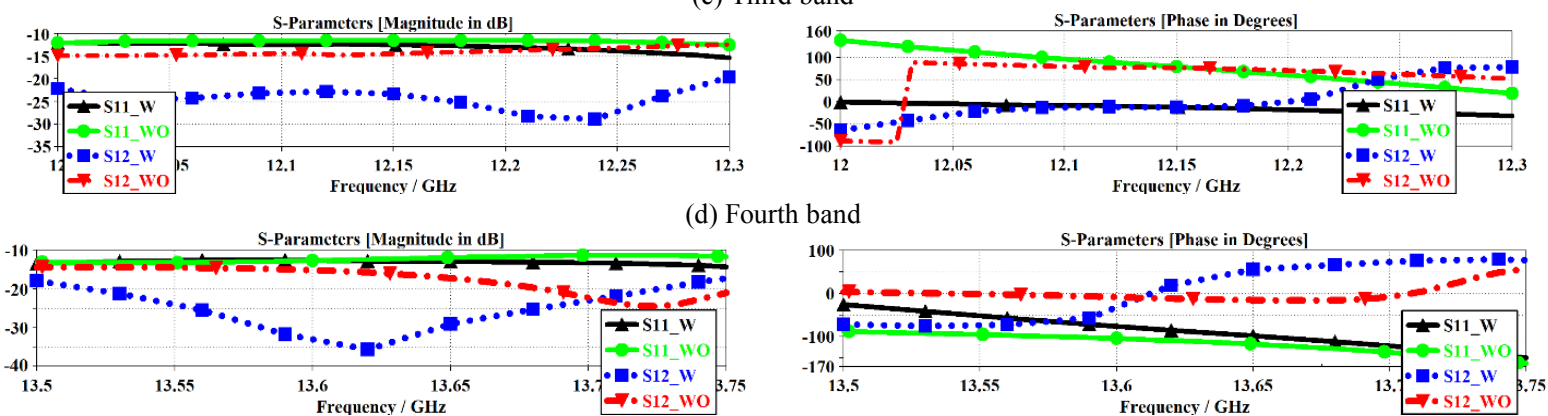

(e) Fifth band
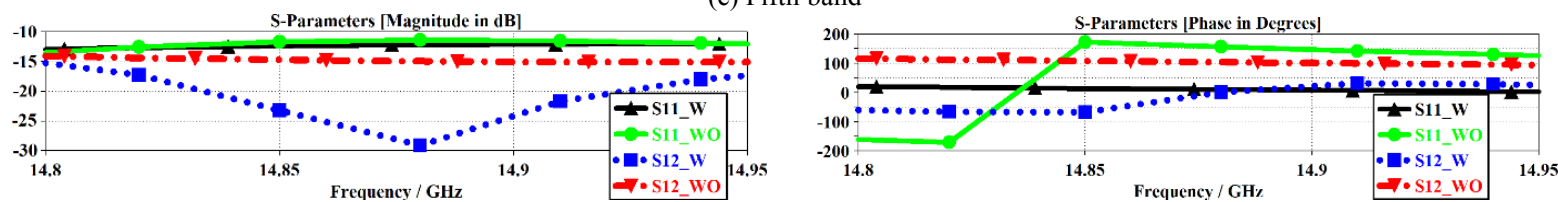

(f) Sixth band

Fig. 2. S-parameter response of the reference and proposed substrate integrated circular waveguide slotted antenna array before and after applying MFIs.

Maximum gain and phase response for the reference and proposed cases are shown in Fig. 3. The radiation characteristics of the proposed structure with and without MFIs are shown in Fig. 4 at $8.7 \mathrm{GHz}, 11.1 \mathrm{GHz}$, and 
13.6 GHz. It is evident that the metal fence isolators greatly improve the radiation gain with negligible effect on the E- and $\mathrm{H}$-plane radiation characteristics.

The above results show that the proposed substrate integrated circular waveguide slotted antenna array exhibits high isolation and operates over a large bandwidth covering $\mathrm{X}$ - to Ku-bands. This type of antenna is suitable for synthetic aperture radar (SAR) applications and multiple input multiple output (MIMO) systems.

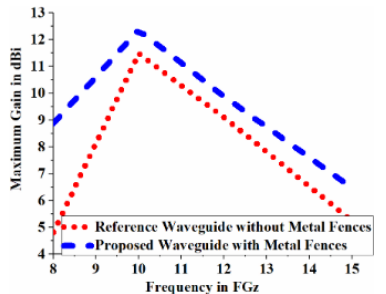

(a)

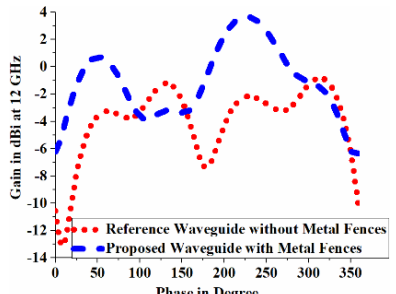

(b)

Fig. 3. (a) Maximum gain over frequency, and (b) phase response at $12 \mathrm{GHz}$.

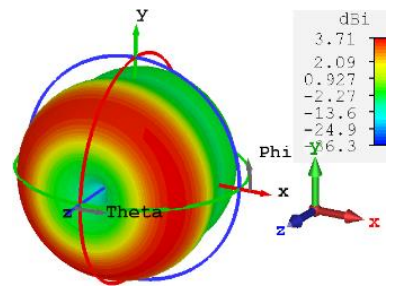

with no MFIs

(a) $8.7 \mathrm{GHz}$

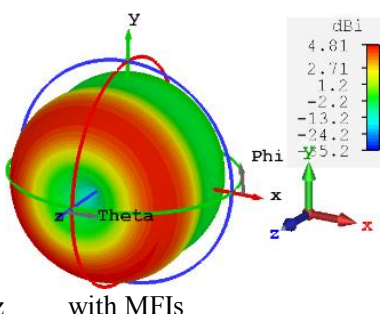

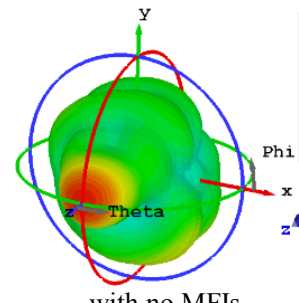

with no MFIs

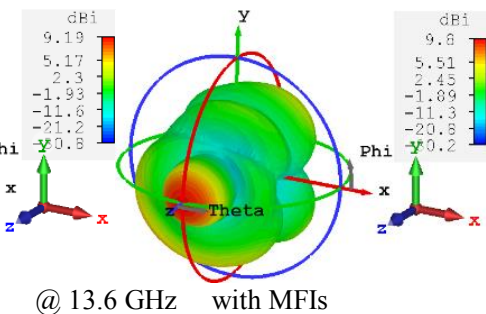

(a) $13.6 \mathrm{GHz}$ with MFIs

Fig. 4. 3-D radiation patterns at $8.7 \mathrm{GHz}$ and $13.6 \mathrm{GHz}$.

\section{CONCLUSION}

It is demonstrated that metamaterial substrate integrated waveguide (SIW) slotted antennas array can operate over a very large frequency range (X-to Ku-band), and with application of metal fence isolators (MFIs) between the slots the mutual coupling between the slots is significantly reduced. Average reduction in isolation over its operating frequency range is approximately $13 \mathrm{~dB}$.

\section{ACKNOWLEDGMENTS}

This work is partially supported by innovation programme under grant agreement H2020-MSCA-ITN-2016 SECRET-722424 and the financial support from the ERDP Yorkshire Innovation 2016-2017, UK.

\section{REFERENCES}

[1] D. Deslandes and K. Wu, "Integrated microstrip and rectangular waveguide in planar form,” IEEE Microw. Wireless Compon. Lett., vol. 11 , no. 2, Feb. 2001, pp. 68-70.

[2] G.-Q. Luo, "Low profile cavity backed antennas based on substrate integrated waveguide technology," in Proc. IEEE APCAP, Singapore, Aug. 2012, pp. 275-276.

[3] Yang Cai, Yingsong Zhang, Can Ding, and Zuping Qian, “A Wideband Multilayer Substrate Integrated Waveguide Cavity-Backed Slot Antenna Array”, IEEE Trans. Antennas Propag., vol. 65, no. 7, July 2017, pp. 3465-3473.

[4] K. Gong, Z.-N. Chen, X.-M. Qing, Z. Song, P. Chen, and W. Hong, "Empirical formula of cavity dominant mode frequency for 60-GHz cavity backed wide slot antenna," IEEE Trans. Antennas Propag., vol. 61, no. 2, Feb. 2013, pp. 1698-1704.

[6] K. Wu, Y. J. Cheng, T. Djerafi, and W. Hong, "Substrate-integrated millimeter-wave and terahertz antenna technology," Proc. IEEE, vol. 100, no. 7, Jul. 2012, pp. 2219-2232.

[5] Y. Zhang, Z.-N. Chen, X.-M. Qing, and W. Hong, "Wideband millimeter-wave substrate integrated waveguide slotted narrow-wall fed cavity antennas,” IEEE Trans. Antennas Propag., vol. 59, no. 5, May 2011, pp. 1488-1496.

[7] L. Xu, L. Li, and W. Zhang, "Study and design of broadband bow-tie slot antenna fed with asymmetric CPW," IEEE Trans. Antennas Propag., vol. 63, no. 2, Feb. 2015, pp. 760-765.

[8] M. Alibakhshi-Kenari, M. Naser-Moghadasi, R. A. Sadeghzadeh, B. S. Virdee and E. Limiti, "Traveling-Wave Antenna Based on Metamaterial Transmission Line Structure for Use in Multiple Wireless Communication Applications," AEUE Elsevier Int. Journal of Electronics and Communications, vol. 70, issue 12, Dec.2016, pp. 1645-1650.

[9] M. Alibakhshi-Kenari, M. Naser-Moghadasi, R. A. Sadeghzadeh, B. S. Virdee and E. Limiti, "New Compact antenna based on simplified CRLH-TL for UWB wireless communication systems," Int. Journal of RF and Microwave Computer-Aided Engineering, vol. 26, issue 3, March 2016, pp. 217-225. 